\title{
Magnetic resonance imaging of intraoral hard and soft tissues using an intraoral coil and FLASH sequences
}

\author{
Tabea Flügge $^{1}$ • Jan-Bernd Hövener ${ }^{2}$ - Ute Ludwig ${ }^{2}$ - Anne-Kathrin Eisenbeiss ${ }^{1}$ • \\ Björn Spittau $^{3}$ - Jürgen Hennig ${ }^{2} \cdot$ Rainer Schmelzeisen $^{1} \cdot$ Katja Nelson $^{1}$
}

Received: 7 July 2015 /Revised: 7 January 2016 / Accepted: 8 January 2016 / Published online: 24 February 2016

(C) The Author(s) 2016. This article is published with open access at Springerlink.com

\begin{abstract}
Objectives To ascertain the feasibility of MRI as a nonionizing protocol for routine dentomaxillofacial diagnostic imaging. Wireless coils were used for MRI of intraoral hard and soft tissues.

Methods FLASH MRI was applied in vivo with a mandible voxel size of $250 \times 250 \times 500 \mathrm{\mu m}^{3}$, FOV of $64 \times 64 \times 28 \mathrm{~mm}^{3}$ and acquisition time of 3:57 min and with a maxilla voxel size of $350 \mu^{3}$ and FOV of $34 \mathrm{~cm}^{3}$ in $6: 40 \mathrm{~min}$. Ex vivo imaging was performed in 4:38 min, with a resolution of $200 \mu^{3}$ and FOV of $36.5 \mathrm{~cm}^{3}$. Cone beam (CB) CT of the mandible and subjects were acquired. MRI was compared to $\mathrm{CBCT}$ and histological sections. Deviations were calculated with intraclass correlation coefficient (ICC) and coefficient of variation $\left(\mathrm{c}_{\mathrm{v}}\right)$.

Results A high congruence between CBCT, MRI and specimens was demonstrated. Hard and soft tissues including dental pulp, periodontium, gingiva, cancellous bone and mandibular canal contents were adequately displayed with MRI.

Conclusions Imaging of select intraoral tissues was achieved using custom MRI protocols with an easily applicable
\end{abstract}

Electronic supplementary material The online version of this article (doi:10.1007/s00330-016-4254-1) contains supplementary material, which is available to authorized users.

Tabea Flügge

tabea.fluegge@uniklinik-freiburg.de

1 Department of Oral and Maxillofacial Surgery, University Medical Center Freiburg, Hugstetter Str. 55, 79106 Freiburg, Germany

2 Medical Physics, Department of Radiology, University Medical Center Freiburg, Breisacher Str. 60, 79106 Freiburg, Germany

3 Institute of Anatomy and Cell Biology, Albert Ludwig University of Freiburg, Albertstr. 17, 79104 Freiburg, Germany intraoral coil in a clinically acceptable acquisition time. Comparison with $\mathrm{CBCT}$ and histological sections helped demonstrate dimensional accuracy of the MR images. The course of the mandibular canal was accurately displayed with $\mathrm{CBCT}$ and MRI.

Key points

- MRI is a clinically available diagnostic tool in dentistry

- Intraoral hard and soft tissues can be imaged with a high resolution with MRI

- The dimensional accuracy of MRI is comparable to cone beam $C T$

Keywords Magnetic resonance imaging · Cone beam computed tomography $\cdot$ Dimensional measurement . Accuracy $\cdot$ Dental Implantation

\section{Introduction}

Medical diagnostic imaging is responsible for close to half (48\%) of human exposure to harmful ionizing radiation, with computed tomography (CT) accounting for roughly half of that amount (24\% of total) [1]. Dental and maxillofacial diagnosis and treatment planning commonly employ different forms of CT, including 64-slice and cone beam CT (CBCT), and studies have been carried out to calculate effective doses for select protocols [2,3]. Wu et al. [4] recently reported that the radiological exposure from 64-slice $\mathrm{CT}$ is associated with a non-negligible lifetime-attributable risk for cancer among the most radiosensitive patients. Despite these risks, CT and CBCT have shown to be useful for the three-dimensional (3D) visualization of the teeth, and vital structures such as the mandibular canal for treatment planning, guided surgery and outcome assessment [5-7]. 
The display of both hard tissue (enamel, dentin, cementum and bone) and soft tissue (gingiva, dental pulp, neurovascular bundle, mucosa, lips and facial tissues) is essential for diagnosis and treatment in various disciplines of dentistry. Some intraoral soft tissues, such as gingiva, are poorly distinguishable in $\mathrm{CT}$ and even more poorly so in $\mathrm{CBCT}$, which is a clinically relevant drawback of these techniques.

The need for non-invasive, non-ionizing diagnostic tools has stimulated research on magnetic resonance imaging (MRI) of the maxillofacial region, but has historically been limited by its comparably low spatial resolution and feasibility concerns, such as lesser availability of equipment, long image acquisition times and high cost. Furthermore, despite a superior display of soft tissues, there is often compromised vizualisation of hard tissues (e.g. enamel and cortical bone) due to a low concentration of free hydrogen in solution.

Some progress in better visualizing select hard tissue components of the dentomaxillofacial complex has been reported. Different approaches for the display of dental hard tissues were documented in vitro using specialized single-point imaging (SPI) [8], ultra-short echo time (UTE) [9], or zero echo time sequences (ZTE) $[9,10]$ to adequately detect caries lesions, cracks/fracture of tooth structure, and pulp morphology. The in vivo application of standard MRI sequences in combination with dedicated intraoral coils allowed imaging of a region of multiple teeth, but focused on internal and surface anatomy for caries detection and overview projections [11-13]. The detailed display of bone, the neurovascular bundle and other soft-tissue components was not feasible [11] or not elaborated on in previous studies [12, 13]. A single modality to comprehensively view the various hard and soft tissues in the maxillofacial region with clinical applicability has not yet been achieved.

The aim of this study was to evaluate the ability of select MRI protocols to display such maxillofacial hard and soft tissues utilizing a wireless, inductively coupled intraoral coil and clinically acceptable acquisition times. The teeth, alveolar bone and surrounding mucosal tissues from the resultant MR images were compared with corresponding $\mathrm{CBCT}$ images and histological sections (where applicable).

\section{Material and methods}

In vivo imaging of two patients (29-year-old female and 35 -year-old male) and ex vivo imaging of a dissected human mandible (provided by the Institute of Anatomy and Cell Biology of Albert-Ludwigs-University, Freiburg, Germany) was approved by the Ethics Committee of the Albert-Ludwigs-University of Freiburg (Application no. 338/13).

\section{Wireless, inductively coupled intraoral coils}

Individually fabricated wireless, inductively coupled intraoral coils as described below were used for imaging of the ex vivo specimen and in vivo imaging (Ludwig et al., Manuscript submitted 2015).

The coils were manufactured from insulated 1-mm diameter copper wire, an adjustable capacitor and crossed diodes. Bending the wire formed two parallel loops with a diameter of $2 \mathrm{~cm}$ at a distance of $1.7 \mathrm{~cm}$. An adjustable ceramic capacitor (range 2.5-10 pF, 250 V) (Johanson Manufacturing, Boonton, NJ, USA,) and crossed diodes BAV 99 (MULTICOMP, Germany) were soldered to the parallel part of the coil. The coils were coated in two layers for insulation: Internally with a protective varnish (PLASTIK 70 Schutzlack, Kontakt Chemie, Iffezheim, Germany) superficially with a selfcuring acrylic die material (Pattern Resin, GC America Inc., Alsip, IL, USA). To adjust the coil's resonance frequency, an access hole to the capacitor was maintained. The radiofrequency of the inductively coupled coil (ICC) was measured (ZVB4 Vector Network Analyzer, Rhode \& Schwarz, Munich, Germany) after intraoral placement and fixation to the tooth surface with silicon putty (Optosil, Heraeus Kulzer, Hanau, Germany).

\section{Imaging}

Imaging was performed with a clinical, whole-body $3 \mathrm{~T}$ MR system (TIM Trio, Siemens, Munich, Germany) equipped with a surface loop receiver coil (4-cm radius) and body transmitter coil. Gradient-echo fast low flip angle shots (FLASH) sequences were applied for in vivo and ex vivo imaging.

\section{In vivo imaging}

For in vivo imaging, a 4-cm loop coil (Siemens) was placed close to the cheek opposite to the intraoral coil. A segment of the left mandible was imaged in 3:57 min, with a volume of $64 \times 64 \times 28 \mathrm{~mm}^{3}\left(115 \mathrm{~cm}^{3}\right)$. FLASH sequences were applied with a voxel size of $250 \times 250 \times 500 \mathrm{~mm}^{3}(\mathrm{TE} / \mathrm{TR}=4.2 / 11 \mathrm{~ms}$, flip angle $\alpha=15^{\circ}$, three averages). The maxilla was imaged with an isotropic resolution of $350 \mu \mathrm{m}$, a field of view (FOV) of $34 \mathrm{~cm}^{3}$ within an acquisition time of $6: 40 \mathrm{~min}$ (TE/ $\mathrm{TR}=4.8 / 12 \mathrm{~ms}$, flip angle $\alpha=15^{\circ}$, five averages). The different FOV chosen for the mandible and the maxilla resulted in different image resolutions. The isotropic resolution of the maxilla allows for reconstruction of all orthogonal slices, whereas the anisotropic resolution of the mandible results in a high resolution of selected orthogonal views.

Subsequently, CBCT imaging of the volunteers was performed (3D Accuitomo 170, Morita, Japan, nominal resolution $250 \mu \mathrm{m}, 90 \mathrm{kV}, 201$ images). 


\section{Ex vivo imaging}

The ex vivo mandible was imaged in 4:38 min using a FLASH MRI sequence with an isotropic resolution of $200 \mathrm{~mm}^{3}$ and a FOV of $39 \times 39 \times 24 \mathrm{~mm}\left(36.5 \mathrm{~cm}^{3}\right)(\mathrm{TE} /$ $\mathrm{TR}=4.3 / 12 \mathrm{~ms}$, two averages, $\alpha=15^{\circ}$ ).

After MRI, CBCT of the dissected jaw (Scanora3D, Soredex, Schutterwald, Germany; nominal resolution $250 \mu \mathrm{m}, 85 \mathrm{kV}, 300$ images) was performed.

\section{Histology}

The dissected human mandible was dehydrated with ascending alcohol series (70-100\% ethanol) and degreased with Xylol (Merck, Germany). The specimen was infiltrated with resin in two stages; firstly with a combination of MMA (Merck, Germany) and dibutyl phthalate $10 \%$ (Fluka, Germany) and secondly with a combination of MMA, dibutyl phthalate $15 \%$ and benzoyl peroxide $3 \%$. After curing resin surplus was cut off with a band-saw (Proxxon) and the specimen was sliced in sections of $500-\mu \mathrm{m}$ thickness with a precision saw (Secotom 50, Struers, Germany). Selected slices were fixed on a microscope slide (Loctite 401, Henkel, Germany and Parafilm ${ }^{\circledR}$, Reichelt Chemietechnik, Germany). Thin sections were produced with a water-cooled rotating grinder (Struers) and abrasive papers (grain 1804000) to a thickness of $80 \mu \mathrm{m}$. Thin sections were stained with azur II, pararosaniline. The respective thin section was measured using a digital microscope (Zeiss, Germany) using the software AxioVision (Zeiss).

\section{Data processing}

Information from CBCT and MRI were available in a DICOM format (Digital Imaging and Communications in Medicine, National Electrical Manufacturers Association, Rosslyn, VA, USA). All data were imported into the software Voxim (IVS Solutions, Germany). An automatic threshold-segmentation was applied and followed by a manual segmentation of both imaging modalities (CBCT and MRI).

With the help of the segmented 3D surfaces the MRI and CBCT data were aligned manually. The alignment was adjusted with the orthogonal views of the images in $\mathrm{x}-, \mathrm{y}-$ and $\mathrm{z}$ directions Figs. 1 and 2.

A representative cut through the tooth axis of the second premolar of the lower left jaw was selected for the measurement of the tooth and its surrounding hard and soft tissues (Fig. 3). The ex vivo specimen was measured in the corresponding slice providing actual measurements for comparison with MRI and CBCT data. Corresponding slices were determined with the following protocol. A millimetre measurement grid was placed on the tooth crown in an axial projection of the CBCT data. The largest diameter in the oral-vestibular dimension and mesial-distal dimension were marked to determine the tooth axis. The identical protocol was applied on the histological specimen. The slice representing the largest orovestibular dimension of the tooth was selected for measurement. The identical slice selection for CBCT and MRI data was ensured by alignment of both datasets in a common coordinate system before slice selection in CBCT.

\section{Measurement of dimensional accuracy of CBCT and MRI in comparison to the histological section}

Reference points were marked in the cross-sectional images produced by $\mathrm{CBCT}$ and MRI for the following measurements: (a) vestibular alveolar limbus, (b) oral alveolar limbus, (c) tooth apex, (d) cranial border of mandibular nerve canal and (e) basal border of the mandibular nerve canal. The measurements were performed by two dentists and were repeated three times with at least $24 \mathrm{~h}$ between the measurements. The following distances were compared between MR and CBCT images: (D1) vestibular alveolar limbus - oral alveolar limbus; (D2) oral alveolar limbus - tooth apex; (D3) vestibular alveolar limbus - tooth apex; (D4) tooth apex - cranial border of nerve canal; (D5) cranial border of nerve - basal border of nerve (Fig. 3)

All point selections were performed in histological crosssections, cross-sectional images of MRI and of CBCT respectively, to provide comparable measurements. The deviations between MRI, CBCT and actual dimensions were calculated with the intraclass correlation coefficient (ICC) including the confidence interval $(\mathrm{CI})$ and coefficient of variation $\left(C_{\mathrm{v}}\right)$. The reliability of measurements regarding the examiner was tested with a Wilcoxon signed ranks test and Friedman's test with regard to the three different time points $(\mathrm{P}<0.05)$. The bias of the imaging modalities was calculated with the difference of the measured distances.

\section{Results}

The presented MR technique allowed the display of cancellous bone, gingiva surrounding the teeth and alveolar bone, mucosa constituting the vestibule and the lip, the periodontal apparatus, the dental pulp and the inferior alveolar nerve ex vivo (Fig. 3) and in vivo (Figs. 4 and 5).

With CBCT, gingiva and mucosa were not displayed. The inferior alveolar nerve was indirectly delineated by its bony boundaries; the dental pulp was visible because of the surrounding dental hard tissues (Fig. 3).

\section{Dimensional accuracy of CBCT and MRI}

Measurements of CBCT and MR ex vivo images had an ICC of 0.993 (CI $0.986-0.997$ ) and $C_{\mathrm{v}}$ of $4.6 \%$. The ICC between 


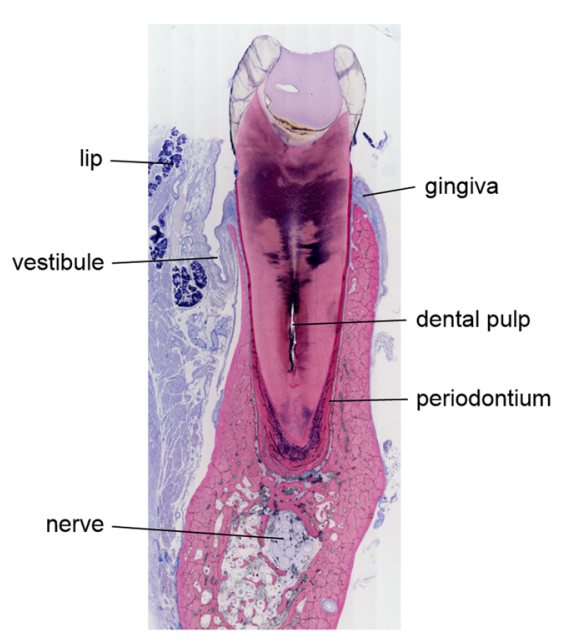

A

Fig. 1 (A) Histological section through the second premolar in the left mandible of an ex vivo human specimen. (B) Section through the magnetic resonance image of the tooth with identical visible structures.

CBCT and the histological thin section was 0.987 (CI 0.974 0.994 ) and the $C_{\mathrm{v}}$ was $6.3 \%$. Measurements of MRI and the histological thin section resulted in an ICC of 0.99 (CI 0.9790.995 ) and a $C_{\mathrm{v}}$ of $5.6 \%$. Measurements of CBCT and MR in

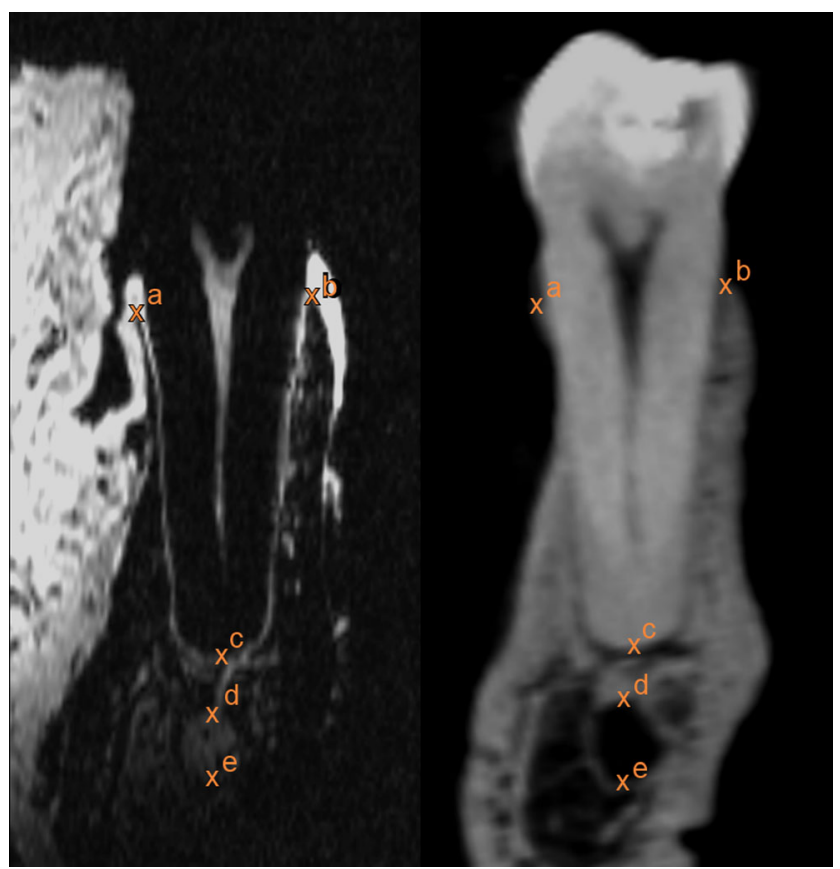

Fig. 2 Section through the magnetic resonance image (left) and CBCT image (right) of the second premolar of the ex vivo lower jaw. The following landmarks are marked in the magnetic resonance image and the cone beam computed tomography image: (a) vestibular alveolar limbus, (b) oral alveolar limbus, (c) tooth apex, (d) cranial border of the mandibular nerve canal and (e) basal border of the mandibular nerve canal vivo had an ICC of 0.99 (CI 0.952-0.997) and a $C_{\mathrm{v}}$ of $5.5 \%$ (see Table 3).

No significant differences were detected between the measurements of Examiner 1 and Examiner 2 regarding all imaging methods: CBCT ex vivo $(\mathrm{p}=0.75)$; MRI ex vivo $(\mathrm{p}=0.96)$; histology $(\mathrm{p}=0.87)$; CBCT in vivo $(\mathrm{p}=1.0)$ and MRI in vivo $(p=0.73)$. The time point was not detected as significant for the measurement results of each method: CBCT ex vivo $(p=0.92)$; MRI ex vivo $(p=0.61)$; histology $(\mathrm{p}=0.5)$; CBCT in vivo $(\mathrm{p}=0.78)$ and MRI in vivo $(\mathrm{p}=0.7)$ (see Table 4). The mean bias for the measurements in MR and CBCT images and histological sections were calculated with the difference of the mean measurement values. The difference between the CBCT and MRI of the ex vivo specimen was 0.09 (CI $-0.1-0.28$ ), the difference between MRI and the histological section was -0.04 (CI $-0.27-0.19)$ and the difference between CBCT and the histological section was -0.13 (CI -0.39-0.13). For the measurements of in vivo imaging, the difference between CBCT and MRI was 0.49 (CI 0.26$0.73)$.

The mean values of all measurements are listed in Tables 1 and 2 for ex vivo and in vivo imaging, respectively. The relationship between measurements in CBCT, MRI and histological specimen are displayed in Fig. 5.

\section{Discussion}

The display of both hard and soft tissue components of the intraoral anatomy is highly important for diagnostic imaging in dentistry. Routinely applied x-ray techniques (CT and 


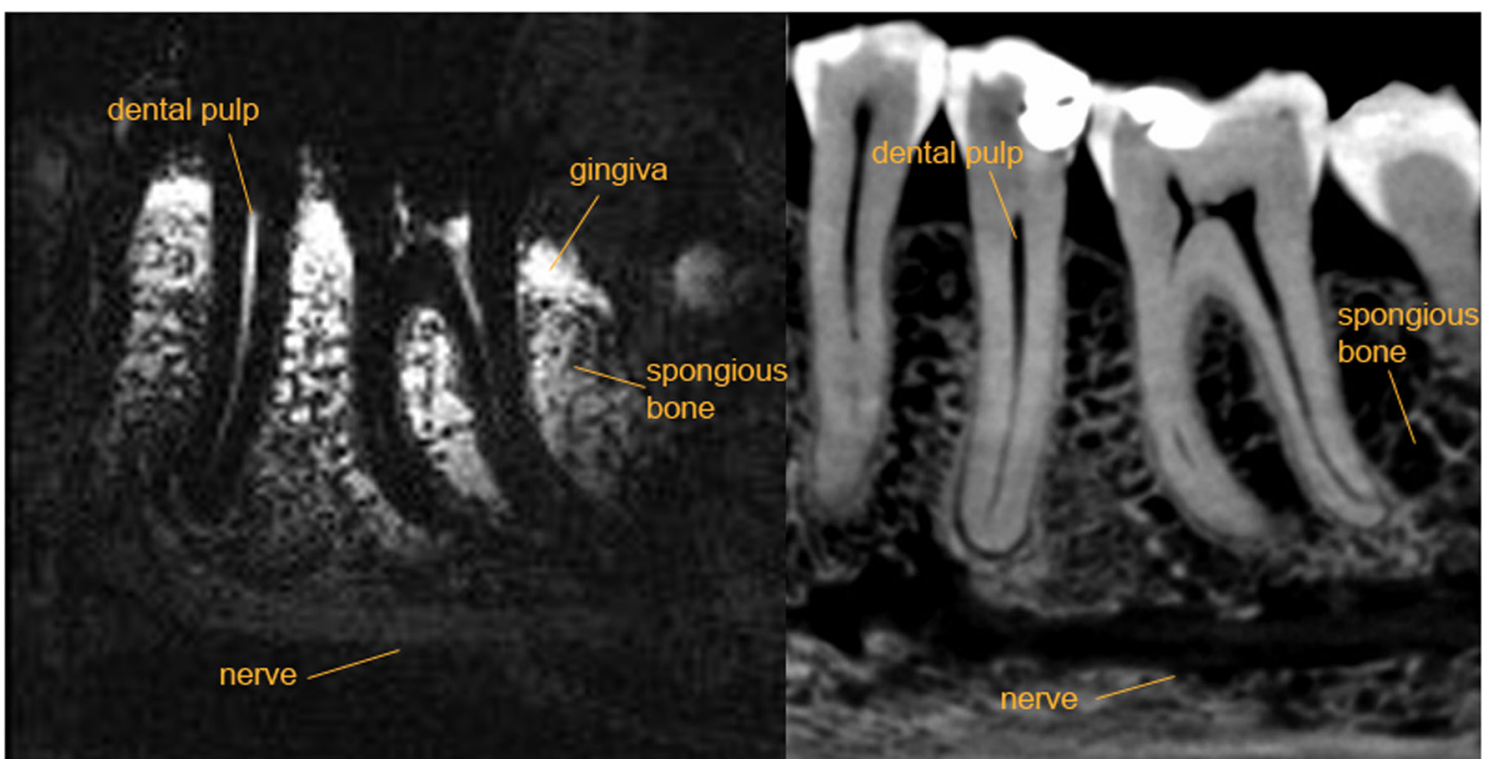

Fig. 3 Sagittal section through the magnetic resonance (MR) images (left) and the cone beam computed tomography (CBCT) images (right) of the ex vivo lower jaw. The dental pulp, gingiva, spongious bone and

CBCT) involve ionizing radiation and are limited in their ability to adequately display intraoral soft tissues. MRI is known for the superior imaging of soft tissues, but has thus far been relatively untested and unfeasible for routine dentomaxillofacial diagnosis.

Within this study, a novel method for MRI protocols was applied to yield images of intraoral anatomy with a spatial resolution high enough to differentiate many tissues/structures, short acquisition times, and potential for clinical application. Inductively coupled coils for use with a standard $3 \mathrm{~T}$ system were built for intraoral application and were used on an ex vivo specimen of a human jaw and in vivo. The intraoral coil created an increased signal within a defined FOV to obtain high-resolution images within an acquisition time applicable for clinical routine.

The presented MR technique allowed the display of cancellous bone, gingiva surrounding the teeth, mucosa comprising the vestibule and the lip, much of the periodontal apparatus, the dental pulp and the inferior alveolar nerve. Another unique finding was the ability to visualize individual branches of the inferior alveolar neurovascular bundle projecting from the canal to supply teeth. Furthermore, delineation of the cortical bone was achieved indirectly by differentiating the relatively hyperintense signals of the surrounding soft tissue and the internal cancellous bone with the hypointense, black signal corresponding to the cortical bone. Therefore, essential structures for preoperative imaging diagnostics in dental surgery, especially implant surgery, were identified. With the presented technique, the coronal part of the tooth was not imaged. The registration of MRI data with surface data, derived from optical or conventional tooth impressions, could conceivably allow for the production of a splint for guided implant surgery. inferior alveolar nerve are discerned in the MR image, whereas the dental pulp canal, the bony borders of the inferior alveolar nerve and the spongious bone are delineated with the CBCT

The registration of radiographic and surface data is common practice in guided implant surgery for the production of surgical splints. Previous studies demonstrating the display of coronal tooth structures with MRI may serve as a foundation to facilitate and direct further research regarding implant surgical guide fabrication using MRI.

In vitro imaging of teeth and their different mineralized parts with MRI was demonstrated before using ZTE, UTE sequences at $7 \mathrm{~T}, 9.4 \mathrm{~T}$ [9], $11.4 \mathrm{~T}$ [10] and at $2.35 \mathrm{~T}$ and $9.4 \mathrm{~T}$, respectively, with long scan times $[13,14]$. The high magnetic inductivity and the longer scan times are currently significant obstacles to clinical feasibility. The in vivo utilization of UTE sequences effectively delineated caries from calcified parts of the tooth but required about 25 min for the display of a single tooth [15].

Several authors have previously identified the need for dedicated coils producing high quality in vivo MR images [11-13]. Idiyatullin et al. utilized SWIFT (Sweep Imaging with Fourier Transform) sequences with a very short echo time (TE) for the detection of hard tissues and introduced a non-specified intraoral coil for dental MRI. Calcified parts of the teeth were displayed whereas soft tissues could not be discerned in detail within an unspecified FOV, an acquisition time of $10 \mathrm{~min}$ and a resolution of $\sim 400 \mu \mathrm{m}^{3}$ [13].

With a cable-bound intraoral coil placed between the teeth, Idiyatullin et al. acquired images with an in-plane resolution of $300 \mu^{3}$, a FOV of $12 \mathrm{~cm}^{3}$ within $4.5 \mathrm{~min}$. The large FOV included the teeth of the upper and lower jaw; however, the periapical region, the alveolar bone and the inferior alveolar nerve were not displayed [11].

Tymofiyeva et al. [12] introduced a wired intraoral coil that was used in combination with a contrasting agent consisting 


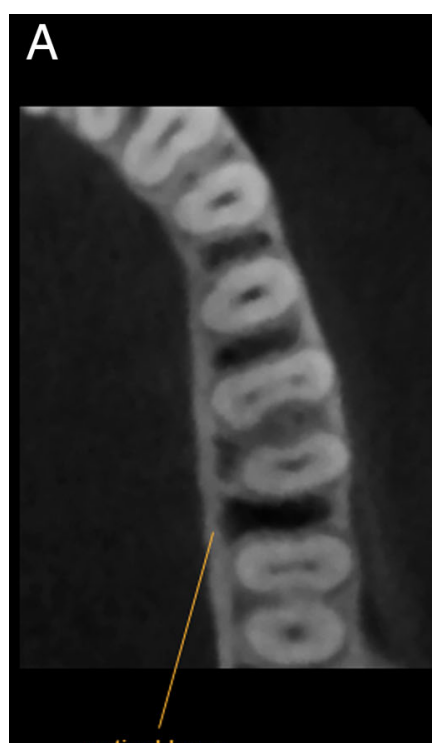

cortical bone

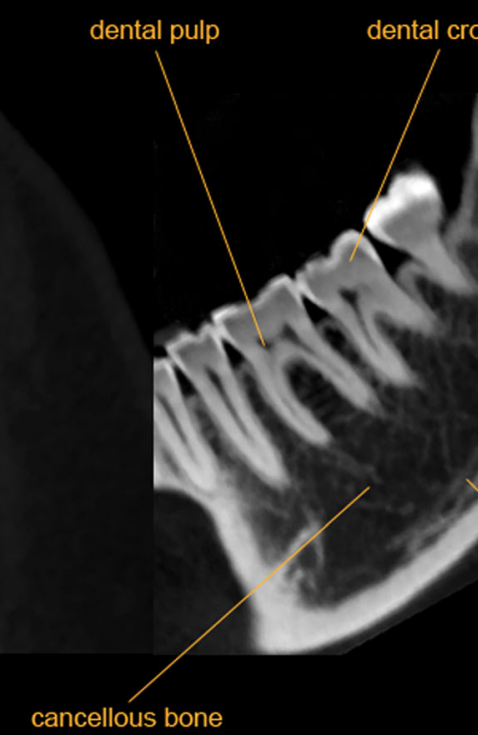

cancellous bone ental crown

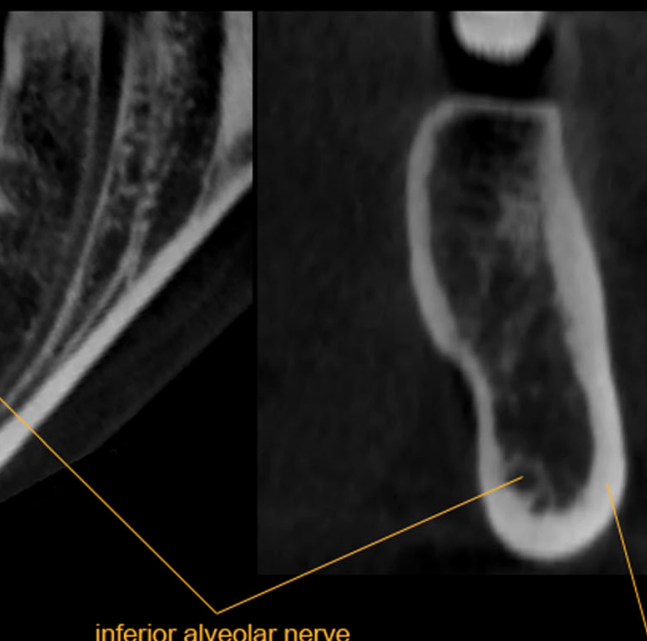

cortical bone

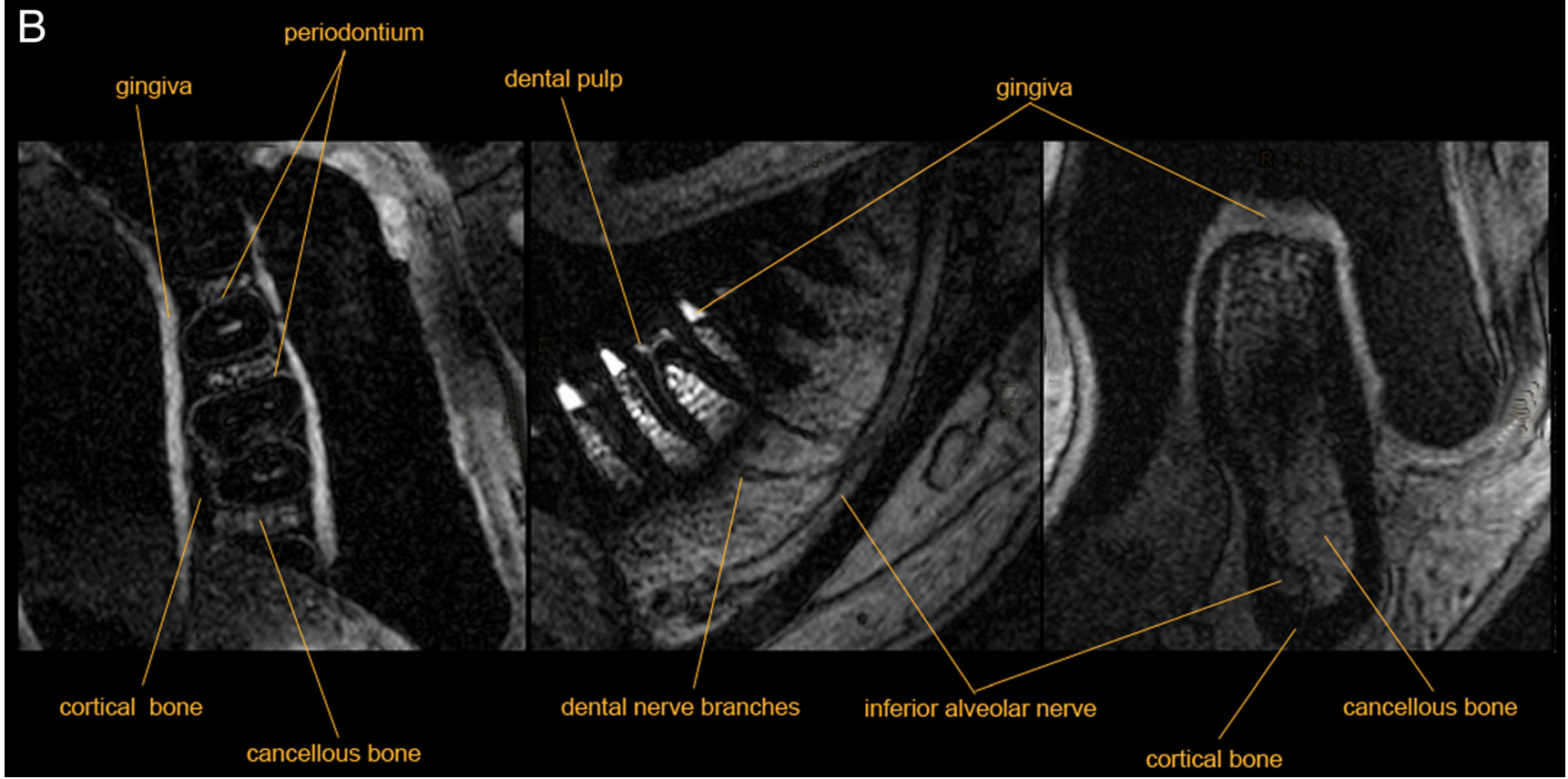

Fig. 4 (B) Axial (left), sagittal (middle) and transverse (right) crosssections of FLASH magnetic resonance imaging of the lower jaw acquired in vivo with an intraoral coil that was inductively coupled to a small loop coil at 3T with the following parameters: $250 \mu \mathrm{m}^{2} \times 500 \mu \mathrm{m}$ resolution, $64 \mathrm{~mm} \times 64 \mathrm{~mm} \times 28 \mathrm{~mm}$ field of view, acquisition time
3:57 min. (A) Axial (left), sagittal (middle) and transverse (right) crosssections of in vivo cone beam computed tomography of the lower jaw (3D Accuitomo 170, Morita, Japan, nominal resolution $250 \mu \mathrm{m}, 90 \mathrm{kV}$, 201 images)

Earlier studies measuring the accuracy of MRI data had a lower image resolution and did not consider in vivo conditions for the comparison of MRI and CT. Furthermore, while comparisons of MR data with that of $\mathrm{CT}$ have been reported, no such comparisons with CBCT specifically have been reported to date. Dimensional accuracy of MRI was demonstrated with the detection of the course of the inferior alveolar nerve in MRI and CT data with the help of grey values [19] and with er resolutions $\left(0.6 \mathrm{~mm}^{3}-1.2 \mathrm{~mm}^{3}\right)$ were achieved. 


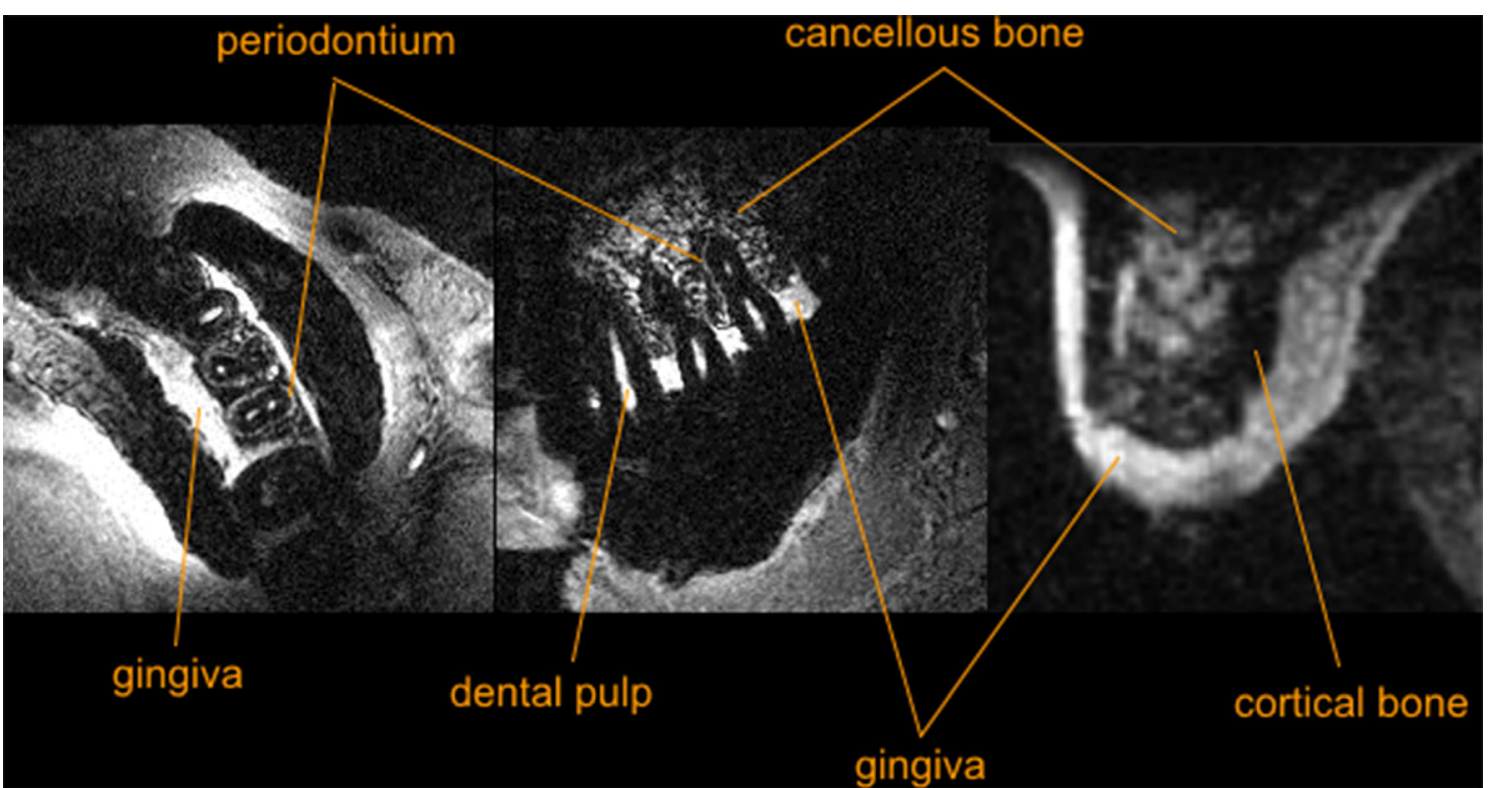

Fig. 5 Axial (left), sagittal (middle) and transverse (right) cross-sections of in vivo FLASH magnetic resonance imaging of the maxilla acquired with a small loop coil at $3 \mathrm{~T}$ and an inductively coupled coil with the following parameters: isotropic resolution of $350 \mu \mathrm{m}$, field of view of $34 \mathrm{~cm}^{3}$, acquisition time of $6: 40 \mathrm{~min}$ (TE/TR $=4.8 / 12 \mathrm{~ms}$, flip angle $\alpha=$ $15^{\circ}$, five averages) the comparison of bony anatomical landmarks of the mandible [20]. In this study, the comparison of MR images with conventional CBCT images and histological sections of the ex vivo specimen showed a high dimensional accuracy of both imaging modalities. Images of the maxilla and the mandible were acquired in vivo; however, the images of the mandible were used for accuracy analysis. Similar anatomical landmarks of the mandible were identified in in vivo and ex vivo imaging data for comparability of measurements. In clinical use, the identification of the inferior alveolar nerve is a major consideration for guided implant surgery.

Although different reference points were used in CBCT (borders of bony nerve canal; bony limbus alveolaris) and MRI (inferior alveolar nerve borders; gingiva covering limbus alveolaris), the intraclass correlation coefficient showed a high congruence between the imaging modalities and between the histological measurements. The coefficient of variation was smaller for MRI measurements compared to CBCT measurements, demonstrating more precise measurements obtained with MR images. In vivo the measurement bias was higher than ex vivo, with a tendency to longer distances measured in CBCT images than in MR images.

MRI is still inferior to CT and CBCT in point of display of hard tissues. Specifically, the tooth surface anatomy is not delineated, which is a limitation of the presented MRI protocols. The utilization of an inductively coupled intraoral coil allows for a higher resolution in a defined FOV. However, the spatial resolutions currently reached are lower than the potential spatial resolutions of CT and CBCT. The delineation of fine structures such as bony trabeculae or the periodontal ligament may thus be complicated. The field with an enhanced signal created by the intraoral coil has to include the tooth apex and mandibular canal to prevent a decreased signal with lesser visibility of the apical periodontal ligament, as observed in Fig. 3. The application of the intraoral coil in more subjects and with relation to various indications has to be conducted to further elaborate on sensitivity and specificity of the presented protocols.

In this study, measurements were reported for CBCT and MR images of the mandible. With regard to dimensional accuracy, the future assessment of MRI of the maxilla should include its comparison with CBCT images.

The inferior alveolar neurovascular bundle was clearly identified in all MR cross-sectional images. With CBCT, the inferior alveolar nerve was discerned by its bony boundaries. While it is conventionally understood that the radiolucent (or low attenuation) signal within the canal boundaries corresponds to the canal's contents in CT/CBCT, the ability of MR to yield a relatively hyperintense signal for the canal contents could potentially have superior diagnostic abilities to $\mathrm{CT} / \mathrm{CBCT}$ to detect abnormalities or pathologies within the tissues comprising the neurovascular bundle itself. An example of this is potential identification of a neuroma, haematoma or physical nerve damage in the absence of gross changes to the bone boundaries of the canal. Conventional MRI protocols may already demonstrate utility for some mandibular canal diagnostic tasks, but protocols such as those presented in this study may be a step toward optimization of diagnostic utility in cases where multiple aspects of dental/ intraoral tissues must be assessed together (such as tooth apex and mandibular canal). 
Other areas of potential future study include the assessment of longitudinal changes of soft and hard tissue over the course of dental therapy. MR shows potential to be a useful modality to study inflammatory or pathological processes of the periodontium and/or bone by assessing pre-treatment, during treatment and post-treatment states, given that visualization of the gingiva/ periodontal apparatus was among the strengths of this study.

\section{Conclusions}

MRI demonstrates potential utility in imaging intraoral hard and soft tissues, and complies with the undisputed aim of dose reduction in medical diagnostic imaging. The presented intraoral coil can easily be applied, and with the FLASH sequences described in our study, adequate visualization of various tissues/structures crucial to oral and maxillofacial diagnosis and treatment planning was achieved in reasonable acquisition times. Sensitivity and specificity of this modality compared with other commonly used methods is yet to be assessed for select diagnostic tasks, but our study demonstrated a high level of dimensional accuracy and congruence compared to $\mathrm{CBCT}$ and histological specimens. The advantages of MRI point towards a broadened application and further optimization of MRI in dentistry.

Acknowledgements The authors wish to thank Dipl.-Math. Gerda Siebert for her help with statistical analysis of data. The scientific guarantor of this publication is Prof. Katja Nelson. The authors of this manuscript declare no relationships with any companies whose products or services may be related to the subject matter of the article. The authors state that this work has not received any funding. JBH wishes to acknowledge support by the German Research Foundation (DFG, Emmy Noether Programme, HO 4604/2-1).

Institutional Review Board approval was obtained. Written informed consent was obtained from all subjects (patients) in this study. Methodology: experimental, performed at one institution.

Open Access This article is distributed under the terms of the Creative Commons Attribution-NonCommercial 4.0 International License (http:// creativecommons.org/licenses/by-nc/4.0/), which permits any noncommercial use, distribution, and reproduction in any medium, provided you give appropriate credit to the original author(s) and the source, provide a link to the Creative Commons license, and indicate if changes were made.

\section{References}

1. National Council on Radiation Protection and Measurements (2009) Ionizing radiation exposure of the population of the United States. Bethesda, Md: National Council on Radiation Protection and Measurements

2. Araki K, Maki K, Seki K, Sakamaki K, Harata Y, Sakaino R et al (2004) Characteristics of a newly developed dentomaxillofacial Xray cone beam CT scanner (CB MercuRay): system configuration and physical properties. Dentomaxillofac Radiol 33(1):51-9, Epub 2004/05/14. PubMed

3. Ludlow JB, Ivanovic M (2008) Comparative dosimetry of dental CBCT devices and 64-slice CT for oral and maxillofacial radiology. Oral Surg Oral Med Oral Pathol Oral Radiol Endod 106(1):106-14, Epub 2008/05/28. PubMed

4. Wu TH, Lin WC, Chen WK, Chang YC, Hwang JJ (2015) Predicting cancer risks from dental computed tomography. J Dent Res 94(1):27-35, PubMed

5. Markiewicz MR, Bell RB (2011) Modern concepts in computerassisted craniomaxillofacial reconstruction. Curr Opin Otolaryngol Head Neck Surg 19(4):295-301, PubMed

6. de Almeida EO, Pellizzer EP, Goiatto MC, Margonar R, Rocha EP, Freitas AC Jr et al (2010) Computer-guided surgery in implantology: review of basic concepts. J Craniofac Surg 21(6):1917-21

7. Mezger U, Jendrewski C, Bartels M (2013) Navigation in surgery. Langenbeck's Arch Surg/ Deut Ges Chirurgie 398(4):501-14

8. Gruwel MLH, Latta P, Tanasiewicz M, Volotovskyy V, Šramek M, Tomanek B (2007) MR imaging of teeth using a silent single point imaging technique. Appl Phys A 88(4):763-7

9. Hovener JB, Zwick S, Leupold J, Eisenbeibeta AK, Scheifele C, Schellenberger F et al (2012) Dental MRI: imaging of soft and solid components without ionizing radiation. J Magn Reson Imaging: JMRI 36(4):841-6

10. Weiger M, Pruessmann KP, Bracher AK, Kohler S, Lehmann V, Wolfram U et al (2012) High-resolution ZTE imaging of human teeth. NMR Biomed 25(10):1144-51

11. Idiyatullin D, Corum CA, Nixdorf DR, Garwood M (2013) Intraoral approach for imaging teeth using the transverse B field components of an occlusally oriented loop coil. Magn Reson Med: Off J Soc Magn Reson Med / Soc Magn Reson Med. doi:10.1002/ mrm.24893

12. Tymofiyeva O, Rottner K, Gareis D, Boldt J, Schmid F, Lopez M et al (2008) In vivo MRI-based dental impression using an intraoral RF receiver coil. Concepts Magn Reson Part B: Magn Reson Eng 33(4):244-51

13. Idiyatullin D, Corum C, Moeller S, Prasad HS, Garwood M, Nixdorf DR (2011) Dental magnetic resonance imaging: making the invisible visible. J Endod 37(6):745-52

14. Sustercic D, Sersa I (2012) Human tooth pulp anatomy visualization by 3D magnetic resonance microscopy. Radiol Oncol 46(1):1-7

15. Bracher AK, Hofmann C, Bornstedt A, Boujraf S, Hell E, Ulrici J et al (2011) Feasibility of ultra-short echo time (UTE) magnetic resonance imaging for identification of carious lesions. Magn Reson Med: Off J Soc Magn Reson Med / Soc Magn Reson Med 66(2): $538-45$

16. Tymofiyeva O, Rottner K, Jakob PM, Richter EJ, Proff P (2010) Three-dimensional localization of impacted teeth using magnetic resonance imaging. Clin Oral Investig 14(2):169-76

17. Olt S, Jakob PM (2004) Contrast-enhanced dental MRI for visualization of the teeth and jaw. Magn Reson Med: Off J Soc Magn Reson Med / Soc Magn Reson Med 52(1):174-6

18. Gahleitner A, Nasel C, Schick S, Bernhart T, Mailath G, Dorffner S et al (1998) Dental magnetic resonance tomography (dental MRI) as a method for imaging maxillo-mandibular tooth retention structures. RoFo: Fortschr Gebiete Rontgenstrahlen Nuklearmedizin 169(4):424-8

19. Eggers G, Rieker M, Fiebach J, Kress B, Dickhaus H, Hassfeld S (2005) Geometric accuracy of magnetic resonance imaging of the mandibular nerve. Dentomaxillofac Radiol 34(5):285-91

20. Goto TK, Nishida S, Nakamura Y, Tokumori K, Nakamura Y, Kobayashi K et al (2007) The accuracy of 3-dimensional magnetic resonance $3 \mathrm{D}$ vibe images of the mandible: an in vitro comparison of magnetic resonance imaging and computed tomography. Oral Surg Oral Med Oral Pathol Oral Radiol Endod 103(4):550-9 